\title{
Preoperative percutaneous carotid artery recanalization in a patient with aortic dissection type A
}

\author{
Hug Aubin ${ }^{1}$, Egzon Memedi $^{1}$, Arash Mehdiani ${ }^{1}$, Artur Lichtenberg ${ }^{1}$, and Alexander Albert ${ }^{2}$ \\ ${ }^{1}$ Heinrich-Heine-University Düsseldorf \\ ${ }^{2}$ Hospital Dortmund
}

February 28, 2021

\begin{abstract}
Management of patients with acute type A aortic dissection (ATAAD) presenting with cerebral malperfusion due to carotid artery obstruction is still a major challenge and often associated with poor prognosis despite successful surgical aortic repair, due to prolonged cerebral perfusion deficit. Here, we present the first report regarding successful percutaneous recanalization of an internal carotid artery occlusion in the setting of an ATAAD prior to open surgical aortic repair with excellent clinical outcome after three year follow-up, including almost full neurological recovery.
\end{abstract}

\section{Introduction}

Acute type A aortic dissection (ATAAD) is a life-threatening disease that can present with severe neurological deficits such as a hemi symptomatic aor a comatose state caused by cerebral malperfusion (CM). CM due to concomitant dissection of the supra-aortic vessels with carotid artery occlusion is of especially poor prognosis, with up to $40 \%$ in-hospital mortality ${ }^{(1)}$.

Established consensus in the treatment of ATAAD patients is immediate surgery in order to replace the damaged aortic segments, obliterate blood flow in the false lumen and restore blood supply of the brain and visceral organs. Nonetheless, surgical repair for ATAAD in presence of internal carotid artery (ICA) obstruction may often be futile, as patients are of high risk of developing cerebral edema and herniation syndrome, and has recently been shown of fatal prognosis ${ }^{(1)}$.

Here, were present a case of an ATAAD patient with left-sided ICA occlusion, in whom percutaneous carotid recanalization and stenting was performed prior to open aortic surgery in order to restore cerebral perfusion in an interdisciplinary, staged treatment approach.

\section{Case report}

A 60-year-old male patient collapsed and was transferred to the next hospital, where he was admitted under the presumed diagnosis of a major stroke due to a new-onset right-sided hemiparesis and motoric aphasia (NIHSS of 12). As the patient was deemed to be in the therapeutic window, he received a systemic thrombolytic therapy. In the subsequent computed tomographic (CT) scan an ATAAD Debakey type I with concomitant bilateral carotid dissection as well as left ICA occlusion up to the intracranial segments was diagnosed (Fig. 1A) and the patient was urgently transferred to our hospital. The patient arrived in a hemodynamic stable condition, but in a comatose state (GCS of 6). A CT perfusion scan of the brain was performed upon arrival in order to evaluate the effects of the thrombolytic therapy, which revealed a persistent major perfusion deficit of almost the complete left-sided cerebral hemisphere (Fig. 1B) . In an interdisciplinary therapy approach it was decided to immediately perform a percutaneous recanalization of the left carotid artery by the interventional neuroradiologists on-site. Hence, three hours after the index 
event two overlapping 9x50mm carotid stents were placed into the left common carotid artery (CCA) and the ICA via right inguinal access (Fig. 2A), with the proximal end of the stent placed approximately in $4 \mathrm{~cm}$ distance to the aortic arch (Fig. 2B). Post-interventional CT scan showed restored left hemispheric blood flow and no cranial hemorrhage nor infarct demarcation. Immediately afterwards the patient was transferred to the operating room, where now six hours after the index event surgical repair of ATAAD was performed, including an aortic valve, ascending aorta and partial aortic arch replacement with a biologic valved aortic conduit as well as an additional right coronary artery bypass under $28^{\circ} \mathrm{C}$ hypothermic circulatory arrest with antegrade brain perfusion via selective cannulation of the truncus brachiocephalicus and the left CCA. During the complete operation non-invasive neuromonitoring via cerebral oximetry showed no side differences nor relevant decrease from initial values.

After the operation the patient showed persistent right-sided hemiparesis with postoperative cranial CTscan revealing only small ischemic watershed lesions on the left cranial hemisphere. Postoperative course was further complicated by an ischemic colitis with concomitant left-sided hemicolectomy three weeks after the initial operation, most likely caused by a pre-existing stenosis of the visceral arteries. Respiratory weaning was accomplished via tracheostomy and the patient could be transferred to the intermediate care station on the $34^{\text {th }}$ postoperative day, from where he was discharged in cardiopulmonary stable status to a neurologic rehabilitation facility with still persistent but regressive right-sided hemiparesis of the upper extremity as well as motoric aphasia.

Three years later the patient presented himself again at our center due to a cutaneous fistula in the lower third of the sternotomy wound. Patient was in a very good general health status and showed almost full neurological recovery with only persistent weakness of the right arm (modified rankin scale: 1). CT-scan showed a stable aortic repair with a patent carotid stent in the left CCA and ICA (Fig. 3) .

\section{Discussion}

Surgical repair for ATAAD in presence of CM due to carotid artery obstruction may often be dismal, as prolonged cerebral perfusion deficit may lead to a disabling ischemic stroke, with major implications on patient's quality of life or may even be futile, as patients are of high risk of developing cerebral edema and herniation syndrome or major cerebral hemorrhage during surgery. Further, ATAAD patients with CM often present with major neurological symptoms and/or in a comatose state questioning surgical candidacy.

A recent study by Fukuhara et al. reviewed 80 ATAAD patients with $\mathrm{CM}^{(1)}$. Only $74 \%$ of those patients underwent open surgical repair. In-hospital mortality was $40 \%$ in the whole cohort and $19 \%$ in the subgroup of patients who received surgical management, with neurological death being the major cause for mortality in those patients. In-hospital mortality was $100 \%$ in patients with ICA occlusion as compared to $21 \%$ in patients with CCA occlusion. Hence, optimal brain salvage strategies in ATAAD patients with CM - and especially ICA occlusion - are still unclear.

From landmark stroke studies it is known that early recanalization of distal ICA occlusion is beneficial regarding clinical outcome ${ }^{(2)}$. However, endovascular carotid recanalization in the setting of ATAAD is still being reported only anecdotally. Heran et al recently presented the case of an ATAAD patient with right CCA occlusion who underwent successful percutaneous carotid stenting prior to surgical repair with excellent neurological outcome ${ }^{(3)}$. However, to the authors' knowledge, the here presented case is the first report regarding successful percutaneous recanalization of an ICA occlusion in the setting of an ATAAD prior to open surgical aortic repair with excellent clinical outcome after three year follow-up, including almost full neurological recovery.

It is important to note, that the limiting outcome factor in most ATAAD patients with CM is the duration of cerebral perfusion deficit. Hence, a staged approach may be most promising in cases where time to restoration of cerebral perfusion can be significantly shortened by immediate endovascular carotid recanalization as compared to immediate surgical repair.

In conclusion, percutaneous endovascular treatment of carotid occlusion in ATAAD patients presenting with 
$\mathrm{CM}$ in order to restore cerebral perfusion prior to surgical aortic repair may improve clinical outcome and reduce neurologic sequelae in selected patients.

\section{References}

1. Shinichi Fukuhara, Elizabeth L Norton, Neeraj Chaudhary, Nicholas Burris, Suzuna Shiomi, et al.. Type A Aortic Dissection with Cerebral Malperfusion: New Insights. Ann Thorac Surg. 2020 Oct 26;S0003-4975(20)31743-4.

2. Henriette Most, Brigitta Reinhard, Brigitta Gahl, Lars Englberger, Alexander Kadner, et al.. Is surgery in acute aortic dissection type A still contraindicated in the presence of preoperative neurological symptoms? Eur J Cardiothorac Surg. 2015 Dec;48(6):945-50; discussion 950. Albers GW, Marks MP, Kemp S, Christensen S, Tsai JP, et al.; DEFUSE 3 Investigators. Thrombectomy for Stroke at 6 to 16 Hours with Selection by Perfusion Imaging. N Engl J Med. 2018 Feb 22;378(8):708-718.

3. Heran MKS, Balaji N, Cook RC. Novel Percutaneous Treatment of Cerebral Malperfusion Before Surgery for Acute Type A Dissection. Ann Thorac Surg. 2019 Jul;108(1):e15-e17.

\section{Figures}

Fig. 1: Preoperative computed tomography scan. (A) Acute type A aortic dissection (white arrow) with concomitant bilateral carotid dissection as well as left ICA occlusion up to the intracranial segments (dashed arrow). (B) Perfusion scan of the brain with major perfusion deficit of almost the complete left-sided cerebral hemisphere.

Fig. 2: Percutaneous carotid intervention. (A) Digital subtraction angiography after stenting of the left CCA and ICA with normal flow into the ipsilateral cerebral hemisphere (extension of the stented area indicated by white arrows). (B) 3D reconstruction from computed tomography angiography directly after percutaneous carotid recanalization, showing carotid stenting of the left CCA and ICA.

Fig. 3: Selected images from computed tomography scan after three year follow-up. CT-scan three years after ATAAD surgery and preoperative carotid recanalization showed a stable aortic repair with a patent carotid stent in the left CCA and ICA (white arrow).

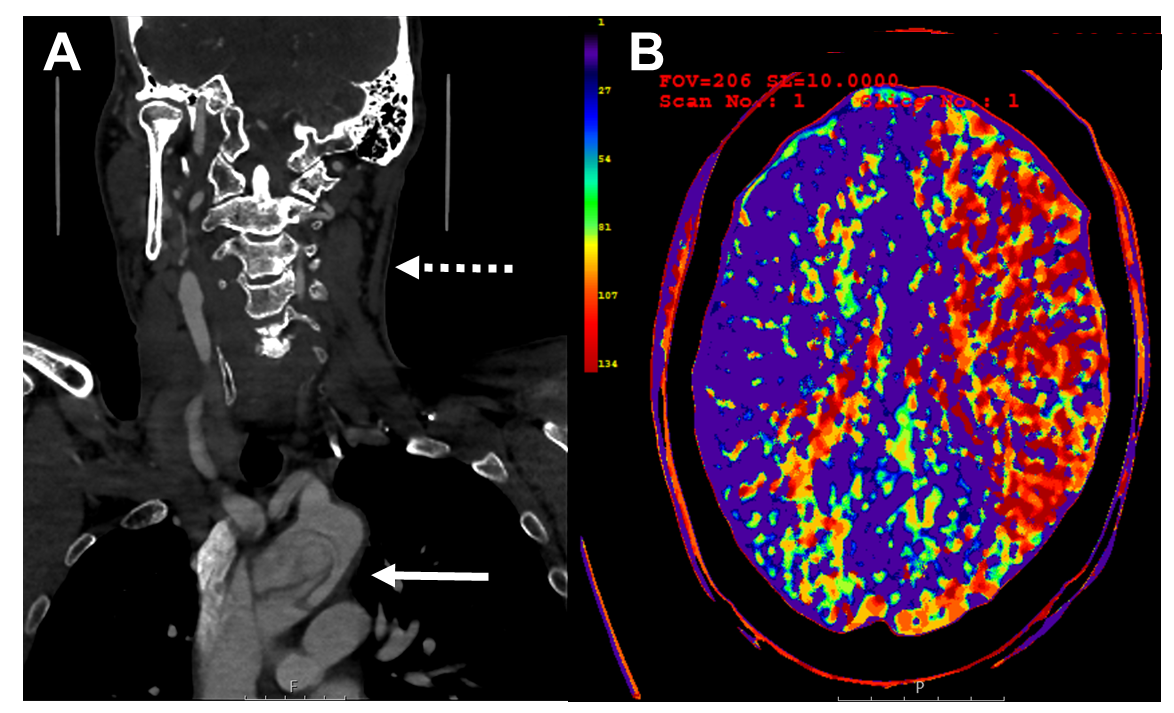



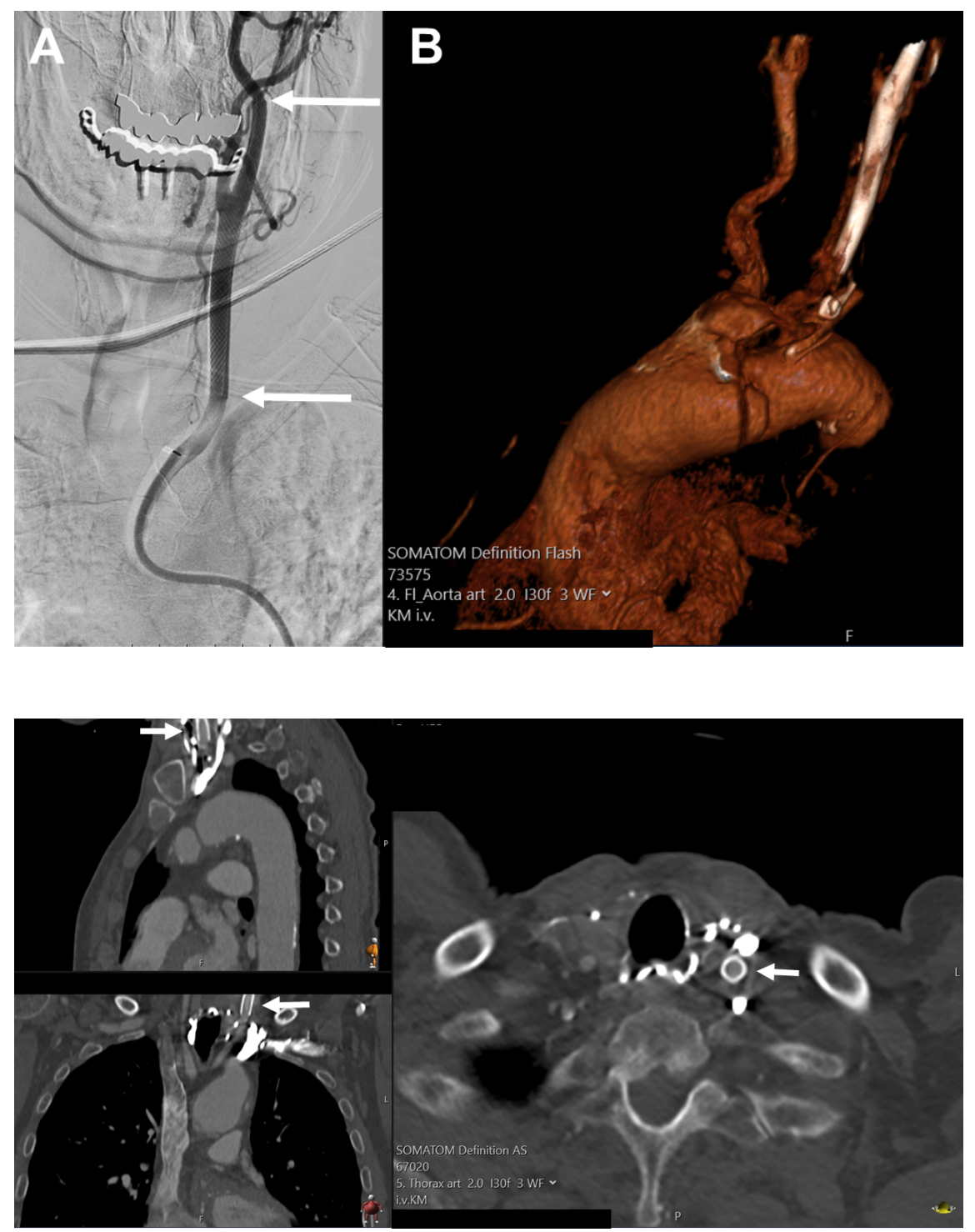